\title{
Cooperative Robots to Observe Moving Targets: A Review
}

\author{
Asif Khan, Bernhard Rinner, and Andrea Cavallaro
}

\begin{abstract}
The deployment of multiple robots for achieving a common goal helps to improve the performance, efficiency and/or robustness in a variety of tasks. In particular, the observation of moving targets is an important multi-robot application that still exhibits numerous open challenges, including the effective coordination of the robots. This paper reviews control techniques for cooperative mobile robots monitoring multiple targets. The simultaneous movement of robots and targets makes this problem particularly interesting, and our review systematically addresses this cooperative multi-robot problem for the first time. We classify and critically discuss the control techniques: Cooperative Multirobot Observation of Multiple Moving Targets (CMOMMT), Cooperative Search, Acquisition, and Track (CSAT), Cooperative Tracking (CT) and Multi-robot Pursuit Evasion (MPE). We also identify the five major elements that characterize this problem, namely the coordination method, the environment, the target, the robot and its sensor(s). These elements are used to systematically analyze the control techniques. The majority of the studied work is based on simulation and lab studies, which may not accurately reflect real-world operational conditions. Importantly, while our systematic analysis is focused on multi-target observation, our proposed classification is useful also for related multi-robot applications.
\end{abstract}

Index Terms-Multi-robot system, cooperative mobile robots, cooperative observation, surveillance, motion planning.

\section{INTRODUCTION}

Cooperative mobile robots for observing multiple moving targets are desirable for a wide range of applications, including search operations [1], sports coverage [2], crowd and social movement monitoring [3], [4], surveillance [5] and wildlife research [6], [7]. Robots, generally equipped with sensors with limited fields of view (FOV), and targets move simultaneously thus creating very challenging dynamic environments for target observation (Fig. 1). A key research task is visibility maximization, i.e. planning the motion of the robots to maximize the number of moving targets under observation [8].

Examples of robotic platforms include Unmanned Ground Vehicles (UGVs), Unmanned Aerial Vehicles (UAVs) and Unmanned Underwater Vehicles (UUVs), which are equipped with sensing, processing, and communication capabilities (Fig. 2). The developments in UGVs and wireless mobile sensor nodes motivate the use of cooperative mobile robots for observing moving targets [9], [10]. Limitations of UGVs include a smaller FOV and movements restricted on a $2 \mathrm{D}$ surface. Recent advancements in UAVs [11], [12] have made

A. Khan is currently with the Ghulam Ishaq Khan Institute of Engineering Sciences and Technology, Topi 23460, Khyber Pakhtunkhwa, Pakistan. He performed this research while he was working with Alpen-Adria-Universität Klagenfurt, Klagenfurt, Austria and Queen Mary University of London, London, UK.

B. Rinner is with the Institute of Networked and Embedded Systems, AlpenAdria-Universität Klagenfurt and Lakeside Labs, Klagenfurt 9020, Austria.

A. Cavallaro is with the Centre for Intelligent Sensing, Queen Mary University of London, London E1 4NS, UK

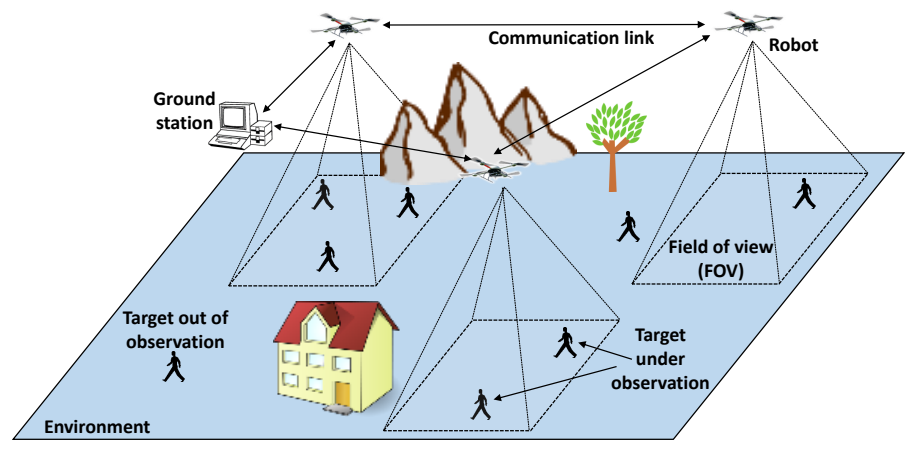

Fig. 1: Illustrative example of cooperative mobile robots for observing multiple mobile targets. Three robots are observing six out of eight targets in a given environment. The coordination may be centralized on the ground station or distributed on the autonomous robots.

it possible to develop approaches for aerial observation of ground moving targets [13], [14]. UAVs, and in particular micro-UAVs with rotors, can fly at low altitudes, hover at a specific point in the environment and move with agility at high speeds [15]. However, the small size of micro-UAVs limits their payload capabilities. The use of UUVs is more difficult due to the additional challenges including underwater transmission of signals (GPS, radio, acoustic) and water currents affecting mobility [16]-[18]. While deployment of UUVs for data gathering is progressing considerably, only few works exist on underwater cooperative UUVs for observing multiple mobile underwater targets [19].

Certain applications of observing multiple moving targets require a team of heterogeneous robots, such as UGVs and UAVs that work together to complete a mission [20], [21], [22]. In such applications, UAVs may act as supervisory agents that detect evading targets but cannot capture them. UGVs then use the information from the UAVs to approach the evading targets and closely observe/capture them.

While a number of reviews on related topics have been published [21], [23]-[32], to the best of our knowledge, our paper is the first overview on multi-robot systems where the robots as well as the targets move in a given environment. Review papers on mobile robots [25]-[27] have focused on the classification and explanation of motion planning approaches for a single robot to explore a region. A system of cooperative mobile robots requires approaches that combine coordination and motion planning [28]-[31]. Existing reviews on cooperative mobile robots encompass tasks without targets, such as collision avoidance, area coverage, map making and marching [31], or with static targets, such as foraging and landmine detection [29]-[31]. Farinelli et al. [23] presented a taxonomy of multi-robot system approaches that classifies them based on 


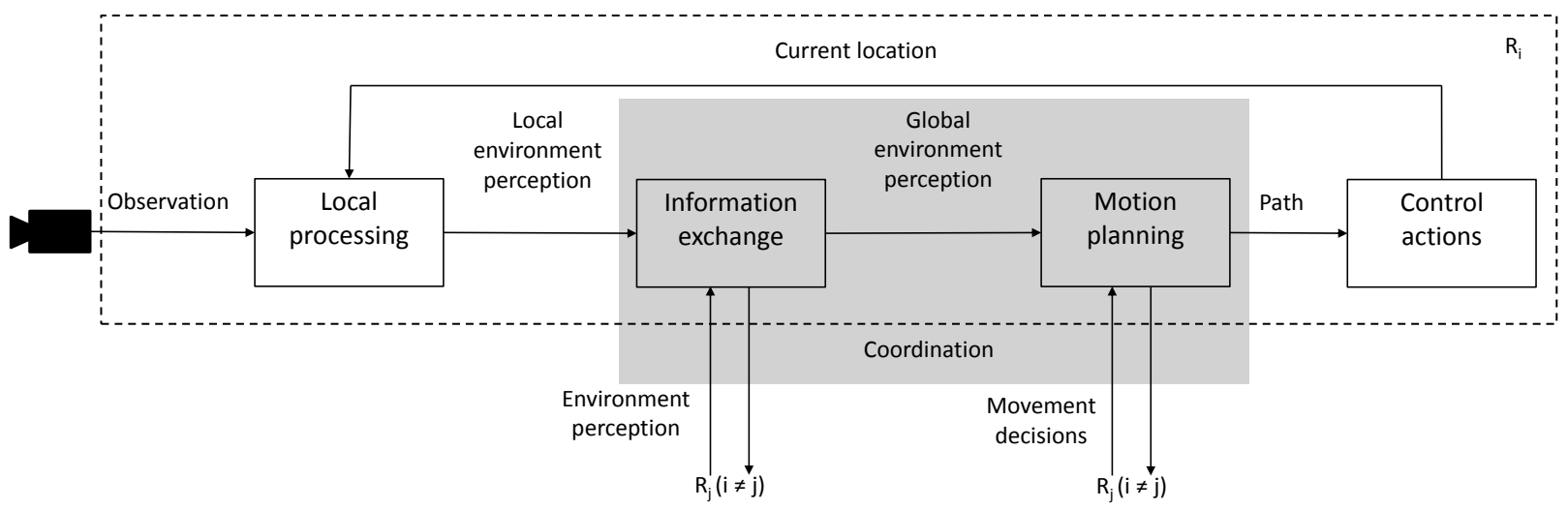

Fig. 2: Information flow and processing steps for cooperative mobile robots. A robot $R_{i}$ observes the targets in the environment and derives local information about the environment and the targets. This local information is exchanged with other robots to derive global information, which serves as key input for the decision-making to plan robot movements. The coordination (information exchange and/or motion planning) can be either centralized or distributed.

coordination and system dimensions. Chen et al. [21] classified approaches for coordination between UAVs and UGVs from an optimization perspective. Only coordination aspects of multirobot systems were covered in [23], [24], whereas the use and benefits of a cloud infrastructure to support the operations of coordinating robots (cloud robotics) were discussed in [24]. A recent and closely related review [32] covers the broad topic of target detection and tracking. So far no review has covered in detail the approaches for cooperative mobile robots equipped with sensing, processing and communication capabilities for observing multiple moving targets. This review fills this gap and narrows the scope of [32]. Note, however, that this review does not include multi-robot systems for observing a single moving target [33]-[37] or multi-target tracking on image sequences [38], [39].

The functionality of cooperative mobile robots depends on five main factors, namely the environment, the target, the robot itself, the sensor(s) onboard the robot and the robot coordination method. We study the main characteristics and effects of these factors on the functionality of cooperative robots and their objectives of observing moving targets. We further group existing approaches based on four major control techniques, namely Cooperative Tracking (CT), where the objective is to persistently track moving targets; Cooperative Multi-robot Observation of Multiple Moving Targets (CMOMMT) [9], which aims to increase the collective time of observation for all targets; Cooperative Search, Acquisition, and Track (CSAT) [13], which alternates search and track of moving targets; and Multi-robot Pursuit Evasion (MPE) [20], whose objective is to capture evasive targets. Table I summarizes these factors as well as the control techniques for observation of moving targets that we discuss in this paper.

The rest of the paper is organized as follows. Section II formulates the problem of cooperative mobile robots observing moving targets. Section III discusses the five key factors affecting the problem. Next, Section IV presents and compares the four major control techniques of observing moving targets using multiple mobile robots. Finally, Section V concludes the paper and presents directions of future research.

\section{PRoblem FORMULATION}

Let a set of $N$ targets $\mathbf{T}=\left\{T_{1}, T_{2}, \ldots, T_{N}\right\}$, whose initial locations and number may be unknown, move independently in a given environment. The state of the $j^{\text {th }}$ target $T_{j}$ at time $t$ is

$$
\mathbf{x}_{j}^{t}=\left(x_{j}, y_{j}, z_{j}\right)^{t},
$$

where $\left(x_{j}, y_{j}, z_{j}\right)^{t}$ denotes the coordinates in the 3D space. In order to observe the moving targets, a set of $M$ robots $\mathbf{R}=\left\{R_{1}, R_{2}, \ldots, R_{M}\right\}$ moves in a given environment $\Omega$. The state of robot $R_{i}$ at time $t$ is

$$
\mathbf{y}_{i}^{t}=\left(x_{i}, y_{i}, z_{i}\right)^{t},
$$

where $\left(x_{i}, y_{i}, z_{i}\right)^{t}$ denotes the position of $R_{i}$ in the environment, which is known to the robot $R_{i}$. In addition to location coordinates, the state vectors of targets and robots may also include information about their velocity, acceleration and orientation. In particular, knowing the orientation is important for robots with directional sensors.

The problem is to decide the motion plans for the robots in order to increase the number of targets under observation by at least one robot. The knowledge of the total number of targets $N$ moving in the environment at a given time influences the cooperative observation process. If $N$ is given and constant for the whole mission, the robots can decide whether to search for unobserved targets or to continue observing already detected targets. If $N$ is unknown, it is not possible to determine when all targets are under observation. Thus, motion planning needs to consider searching unobserved targets throughout the mission. The problem becomes more challenging when the total number of targets $N$ dynamically changes because new targets appear in the environment, some of the existing targets disappear or targets re-enter the environment [7]. In decentralized and distributed multi-robot systems the information about the total and the current number of targets under observation might vary among the robots. Such an inconsistency may be generated by the limitations in local sensing and processing as well as communication, and makes motion planning even more challenging. 
TABLE I: Summary of related work for cooperative observation of multiple moving targets. C: Centralized, Ca: Camera, Co: Cooperative, Cmb: Combination of UAV and UGV, D: Distributed, Dc: Decentralized, DE: Detection Error (False detection and miss detection), Di: Directional, Dt: Deterministic, E: Evasive, Err.: Error, Idt.: Identification, K: Known, M: Number of robots, Mod.: Model, N: Number of targets, Nc: Non-cooperative, Ns: Noise, Om: Omnidirectional, Pr: Probabilistic, Ra: Range, S: Structured, Spc.: Space, Str.: Structure, U: Unstructured, Un: Unknown.

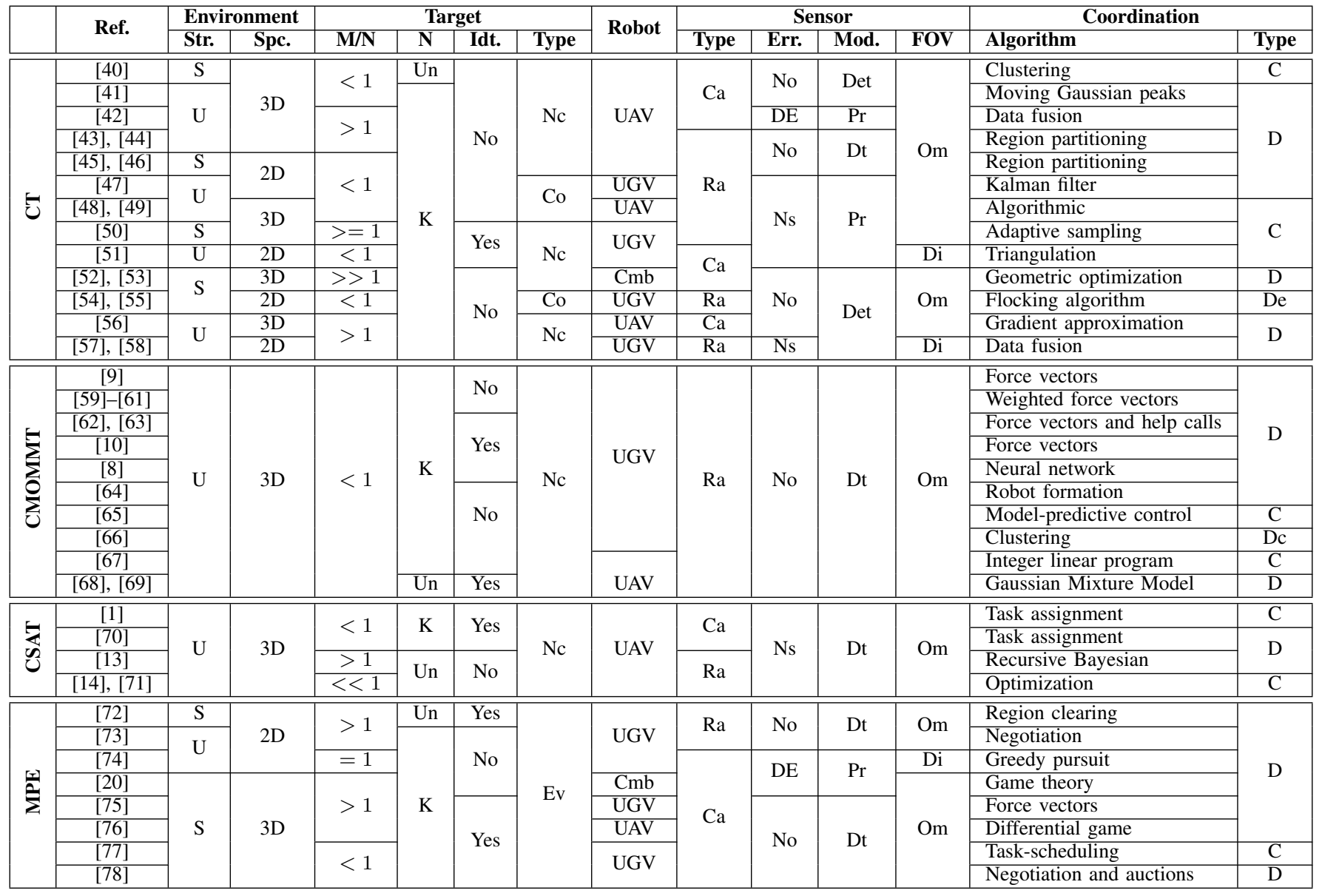

In case of platforms with local processing power, each robot iteratively executes four actions (Fig. 2): taking observations and processing the data locally; exchanging information with other robots (and the ground station); planning its motion; and generating control actions to execute its physical motion according to the plan. Once the robots have moved, the current location information and the new observation from the sensor initiate a new iteration of this processing cycle. Note that this process is different from traditional motion planning and coordination where the robots typically compute shortest paths from their known initial locations to known goal locations [27]. In our problem, the goal locations are the positions of the targets, which are unknown and dynamic.

Although the initial attributes of the multi-robot system may be known, the changing positions of both the targets to be observed and the robots make it difficult to determine the goal representation. Therefore, motion planning and coordination strategies that consider task execution as the transition of the system attributes from an initial representation to the goal representation [79] do not address in their original form the multi-target observation problem addressed in this survey.

\section{COOPERATIVE MOBILE ROBOTS: FIVE FACTORS}

The problem of observing moving targets with cooperative mobile robots can be characterized by five factors: the environment, the targets, the robots, the sensors and the coordination method. The observation of moving targets also depends on what targets the robots need to observe and for how long they need to observe them. In the following sections, we describe these five factors.

\section{A. Environment}

Robots can operate on the ground, in the air or underwater, and even the observation of an environment without targets is a challenging multi-robot control task [80], [81]. In this section, we identify key characteristics of the environment (its representation, structure and evolution) and their effects on our problem. Table II classifies related work based on these key characteristics.

The environment confines the movement of both robots and targets, and affects observations by potentially restricting a sensor's FOV. The environment, which is usually bounded, is represented as a continuous [1], [9], [44], [60] or discrete 
TABLE II: Classification of related work based on key characteristics of the environment (U: Unstructured, S: Structured).

\begin{tabular}{|c|c|c|c|}
\hline \multirow{2}{*}{ Structure } & \multirow{2}{*}{ Evolution } & \multicolumn{2}{|c|}{ Representation } \\
\cline { 3 - 4 } & & $2 \mathrm{D}$ & $3 \mathrm{D}$ \\
\hline \multirow{4}{*}{$\mathrm{U}$} & \multirow{3}{*}{ Static } & {$[57],[58]$} & {$[9],[10],[20],[83],[84]$} \\
\cline { 3 - 4 } & & {$[47],[51]$} & {$[8],[41],[48]$} \\
\cline { 3 - 4 } & & {$[75],[82]$} & {$[56],[59]-[66],[85],[86]$} \\
\cline { 2 - 4 } & Dynamic & {$[74]$} & {$[43],[49],[68]-[71]$} \\
\hline \multirow{2}{*}{$\mathrm{S}$} & \multirow{2}{*}{ Static } & {$[54],[55]$} & {$[45],[46],[50],[52],[53]$} \\
\cline { 3 - 4 } & & {$[72],[87]$} & {$[76]-[78]$} \\
\cline { 2 - 4 } & Dynamic & - & {$[40],[88]$} \\
\hline
\end{tabular}

[82], [83] 2D plane or 3D space. A ground robot may also move on a $2 \mathrm{D}$ surface embedded in a 3D space [60]. A 3D Euclidean space environment facilitates operations of an aerial robot [1], [13], [14], [40], [44], [57], [58], [83]. A region with a regular-shaped boundary such as a circle [60], [65] or rectangle [74] are mostly used for the 2D movement of robots and targets in simulations. Limited research has been conducted using irregular shapes to represent the boundaries of the environment as they introduce extra challenges [68]. Moreover, the terrain of the environment (e.g., planar, irregular or cluttered surface) may limit the mobility of robots.

The structure of an environment may restrict the sensor's observation as well as the movement of targets and robots. This structure is usually represented with a suitable data-structure, such as an occupancy grid map [1], [89] or a Voronoi diagram [77]. A structured environment may consist of a well-defined floor plan known to the robots [40]. Examples of navigation in structured environments include road-following approaches where the aim is to detect movable paths and to navigate them [25]. In structured environments [45], [46], [50], [52], [53], [76]-[78], the location and size of potential obstacles are usually assumed to be known, and allowed and forbidden regions for the movement of targets and robots are explicitly specified. Unstructured environments provide no information about defined paths, boundaries or locations of obstacles [43], [71] and usually represent outdoor environments without a defined map or information about obstacles.

The environment can be static or can evolve dynamically throughout the mission. A static environment consists of unchanging surroundings [9], [47], [57], [62]. In dynamic environments, the movement of obstacles [68], [69], variations in geometry of the environment [40] and variation in terrain [70] continuously change the surroundings of the robots. Operations in dynamic environments require the robots to adapt to changing situations, thus introducing an additional challenge.

\section{B. Target}

Moving targets are typically described by the following three characteristics: type, mobility and representation.

As for type, we can identify cooperative, non-cooperative and evasive targets (Table III). Cooperative targets [5] continuously or occasionally transmit to the robots some information, such as their GPS coordinates or other positional data, thus making localization and observation easier. In most applications, targets are non-cooperative, i.e. they neither send their
TABLE III: Classification of related work based on three different target types and the variability of the number of targets.

\begin{tabular}{|c|c|c|}
\hline \multirow{2}{*}{ Target type } & \multicolumn{2}{|c|}{ Number of targets } \\
\cline { 2 - 3 } & Constant & Variable \\
\hline Cooperative & {$[5],[47],[49],[54],[55]$} & - \\
\hline \multirow{3}{*}{ Non-cooperative } & {$[77],[83],[84]$} & {$[68],[69]$} \\
\cline { 2 - 3 } & {$[1],[8]-[10],[41]-[46]$} & {$[13],[87],[88]$} \\
\cline { 2 - 3 } & {$[59]-[66],[85],[86]$} & {$[40]$} \\
\cline { 2 - 3 } & {$[48],[50],[52],[53],[56],[70]$} & {$[14],[71]$} \\
\hline Evasive & {$[20],[74]-[78]$} & {$[72]$} \\
\hline
\end{tabular}

location information to robots nor hide from observation [9], [10], [13], [14], [62], [63]. Evasive targets can sense the robots and avoid being observed, which makes their observation more difficult [20], [72], [74]-[78].

The mobility of a target depends on its capabilities and is also constrained by the environment. The movements of targets are usually unforeseen and mostly independent of each other. Therefore observing one target does not generally provide useful information about the location or behaviors of other targets. Random walks [47] and linear motion models [14] are the dominant mobility models used in simulation studies. The maximum speed of the target is usually smaller than that of the robots. Most works on cooperative mobile robots for observing moving targets focus on ground targets moving on 2D plane and assume some prior knowledge about the target's mobility. There is only limited research on targets moving in 3D space available, such as aerial targets [49], [85], [90] or underwater targets [19], [86]. Targets moving on an uneven (ground) surface [91] also exhibit 3D movement. However, to the best of our knowledge, 3D movement has not yet been explored for cooperative target observation.

A target can be represented as a point, whose coordinates are determined by the sensor as 2D planar or 3D space coordinates on a pre-defined grid of known size [42], [82]. In addition to the position, the representation of a target can also include constant [13], [57], [60], [62] or variable [68], [69] velocity components. Some approaches combine position and target density information to represent a target [40], [46]. A target can also be represented using a combination of position, velocity and uncertainty/noise components [14], [58], [71].

Most approaches are applicable only for observing a known and constant number of targets moving in the environment [1], [8], [9], [20], [50]-[58], [61]-[63], [74]-[78]. Only few approaches consider the movement of a variable number of targets [68], [72] and account that a target may appear or disappear for certain duration of time [92]. Targets may leave or enter the environment [68] at (usually) known entry or exit points [9], [60]. The lack of prior knowledge (number and location) about the entry/exit points in the environment introduces challenges when observing multiple moving targets [69].

\section{Robot}

A cooperative mobile robot has four main capabilities: sensing, processing, communication, and mobility. 
Robots sense the environment to capture observations and then process these local observations to determine a local perception of the environment and the targets. The processing capability depends on the available resources of the robot. Based on their local perception of the environment, the robots exchange with team members information (e.g. location information, the state of the environment and sensor data) to compute a global perception of the environment. Moreover, to improve their future movement decisions robots may communicate to share their intentions, goals and actions. The movement decisions generate a path for the mobility of the robot, which produces control actions for moving to a new location according to this planned path. The perception of the environment and the movement plan depend on the specific application objective(s). Objectives may include searching the locations of unknown targets and maximizing the localization accuracy of already found targets.

Traditional applications of multi-robot systems are based on homogeneous robots with similar capabilities. Recent developments in robotic applications benefit from a team of heterogeneous robots with different capabilities that increase the performance of the multi-robot system. For example, coordinating UGVs could increase the observation of a region occluded by tall buildings or trees that may not be observed by UAVs only [14], [20], [88]. The operating environment may also demand for heterogeneous robots with different capabilities to explore different parts of the environments [20], [52], [53]. The heterogeneity in robotic platforms introduces the challenge of how to effectively deal with information exchanges and decision making. Moreover, performance guarantees on whether such heterogeneity improve or degrade system performance are needed.

\section{Sensing}

The aggregated area of all the sensors' FOVs is generally much smaller than the area to be monitored. Physical constraints of the robot and the environment may influence the observation and detection capabilities of the sensor the robot is equipped with [51], [74]. Typical sensor types for target observation and detection include vision sensors [40], [76] and range sensors (e.g., radar, sonar, laser scanner) [10], [62], [63], [65] (Table IV).

The observation of a target depends on the type of sensor and its FOV. Let us define the observation of $T_{j}$ at time $t$ by $R_{i}$ with the sensor's FOV $F_{i}$ as

$$
O_{i j}^{t}= \begin{cases}1 & \text { if } \mathbf{y}_{i}^{t} \in F_{i} \\ 0 & \text { otherwise }\end{cases}
$$

A robot can observe more than one target at a time and multiple robots can simultaneously observe the same target. If $O_{i j}^{t}=0$, then the state of $T_{j}$ is unknown to $R_{i}$ at time $t$. However, robots can coordinate with each other by exchanging not only information about themselves but also about the targets under their observation.

In addition to the limited FOV, challenges in sensing include limited sensor performance (e.g. detection errors, noise) and limited observability (e.g. due to occlusions). A deterministic model of a sensor represents a sensor with no errors in reporting the location of a target. In this case, the observation follows the model in Eq. 3. Sensing errors can be modeled with target location uncertainties represented with probability distributions. A probabilistic model includes two types of sensing errors: measurement noise and detection errors. Measurement noise is represented as a probability distribution in the sensor output and models inaccurate estimations of the coordinates of the target location (and its size). Detection errors model false positive detections and miss-detections.

Let $p, q, 1-p$ and $1-q$ denote the probability of detection, the probability of producing a false positive detection, the probability of a miss detection and that of correctly not detecting a target where there are none, respectively. Depending on the target's true presence, $X=1$, or absence, $X=0$, in the FOV $F_{i}$, the detection error based on the observation is one of the following four probabilities, $P($.$) [82]:$

$$
\begin{gathered}
P\left(O_{i j}^{t}=1 \mid X=1\right)=p, P\left(O_{i j}^{t}=0 \mid X=1\right)=1-p \\
P\left(O_{i j}^{t}=1 \mid X=0\right)=q, P\left(O_{i j}^{t}=0 \mid X=0\right)=1-q .
\end{gathered}
$$

Combining the information from multiple sensors mounted either on the same robot or on different robots is desirable for improving the perception of the environment [93], [94]. Information fusion can be employed to improve the robot (self) state estimation with respect to the environment, the targets and the other robots. Information fusion can also improve camera calibration and sensor movement in single-robot applications [95]. Finally, information fusion from heterogeneous sensors, such as a vision sensor and a laser scanner or a vision and a GPS sensor, helps to increase accuracy and robustness against sensing errors as shown for a static sensor network [96]. New techniques are desirable to enhance sensor fusion in both distributed and non-distributed systems.

\section{E. Coordination}

The functionality of cooperative robots [97] depends greatly on their networking capabilities. Coordination among robots is achieved by sharing information to improve the perception of the environment and by jointly performing decision-making for motion planning [98]. This coordination can be centralized, decentralized or distributed (Table V).

Coordination needs reliable networks with guaranteed quality of service (e.g. bandwidth, delay) to cope with connectivity and time-varying network latency of highly mobile and cooperative robots. Wireless communication among the robots is limited and may hinder the information exchange among the robots because of limited bandwidth or temporary loss of connectivity. Moreover, the communication requirements of robots moving in 3D environments [99], [100] are different from those of ground robots.

With a centralized coordination robots exchange information with a central node or ground station that computes and assigns globally optimal plans using task assignment [1], optimization techniques [14], [71], clustering [40], triangulation [51] or scheduling [77]. However, the central node is a single point of failure and might not receive complete and updated information due to sensing and communication limitations. 
TABLE IV: Classification of related work based on sensor type and model (Om: Omni-directional, Di: Directional).

\begin{tabular}{|c|c|c|c|c|c|c|}
\hline \multicolumn{2}{|c|}{} & \multicolumn{3}{|c|}{ Sensor model } \\
\cline { 3 - 7 } \multicolumn{2}{|c|}{} & \multicolumn{2}{|c|}{ Probabilistic } & \multicolumn{2}{c|}{ Deterministic } \\
\hline \multirow{3}{*}{ Sensor type } & \multirow{2}{*}{ Vision } & Om & - & {$[20],[42]$} & {$[13],[70]$} & No error \\
\cline { 2 - 7 } & & Di & {$[51]$} & {$[74],[84]$} & - & {$[40],[41],[54],[55],[75]-[78]$} \\
\cline { 2 - 7 } & \multirow{2}{*}{ Range } & Om & {$[48],[50]$} & - & {$[1],[14],[71]$} & {$[8]-[10],[43],[45],[57],[88]$} \\
\cline { 3 - 7 } & Di & - & - & {$[49],[57],[58]$} & {$[85],[86]$} \\
\hline
\end{tabular}

TABLE V: Classification of related work based on three types of coordination.

\begin{tabular}{|c|c|}
\hline Centralized & {$[1],[3],[14],[40],[65],[77],[83]$} \\
& {$[44],[48]-[51],[71],[84]-[87]$} \\
\hline Decentralized & {$[54],[55],[66],[88]$} \\
\hline \multirow{2}{*}{ Distributed } & {$[8]-[10],[13],[20],[56]-[60]$} \\
& $\begin{array}{c}43],[45],[46],[52],[53],[68],[74],[76],[78] \\
{[41],[42],[47],[61]-[64],[69],[70],[75]}\end{array}$ \\
\hline
\end{tabular}

In fact, communication problems severely affect centrally coordinated robots by isolating (permanently or temporarily) one or more robots from the available global information and decisions.

With decentralized coordination there are multiple leader robots that act as central nodes for smaller groups of robots [54], [55], [66]. Each leader then coordinates with other leaders.

Finally, robots with a sufficient amount of memory and processing power can coordinate in a distributed manner. In distributed coordination each robot decides independently, even with limited available information. Examples for such distributed decision making are based on artificial force vectors [10], [59]-[63], auctions [8], consensus [88], region partitioning [45], [46], data fusion [57], [58] and game theory [20], [76]. Distributed algorithms enable individual robots to operate with partially available information and are therefore only marginally affected by communication problems.

\section{Control Techniques}

In this section we discuss four major control techniques for observing moving targets with multiple mobile robots and identify tasks for which these control techniques are suitable. Table VI summarizes the tasks relevant to the four control techniques, namely Cooperative Tracking; Cooperative Multirobot Observation of Multiple Moving Targets; Cooperative Search, Acquisition and Track; and Multi-robot Pursuit Evasion.

\section{A. Cooperative Tracking (CT)}

Increasing the frequency of target state updates by using sensor observations reduces the uncertainty in target tracking. For this reason, CT aims to minimize the time duration between two consecutive observations of each target [49], [85], [87]. This increase in frequency of sensor observations minimizes the uncertainty over moving target locations and increases the observation of multiple moving targets. The estimated locations of targets are assumed to be known to the robots, which do not search for unknown targets. CT is not matching sensor measurements to target tracks (data association [101]), which is an important problem in radarbased/image-based multi-target tracking. CT is suitable when the number of targets does not considerably exceed the number of robots and there might be the need of close observation of some specific targets, e.g. using security robots [102].

In CT, cooperative paths can be defined for fixed-wing, minimum turn-angle UAVs to increase the frequency of individual observations of a moving target in an uncluttered, outdoor environment [56], [103]. Optimal circular paths can also be designed for fixed-wing, high altitude UAVs to increase the observations of targets in densely populated urban areas [40]. The targets may be partitioned into as many groups as UAVs and the center of each UAV's circular path is updated to maintain the best view of the corresponding group of targets.

When target paths are predefined, a simple strategy of revisiting paths can be designed using moving Gaussian peaks [41]. To best observe targets with known locations, multiple views of the targets may be desirable and can be obtained by observing the target with multiple robots from different view angles [47]. Triangulation-based location estimation consists in moving targets being constantly observed by multiple moving robots [51]. Cooperative paths for the movement of robots can be designed not only to accurately observe and localize the target but also to minimize the energy consumption for the robot movement [104].

Several works track moving targets in outdoor, unstructured, uncluttered and bounded environments. Clustering of robots and a distributed mechanism for coordination are used in [57], [58] to track cooperative targets that transmit signals to the robots. The robots use directional antennas, timeof-arrival and direction-of-arrival measurements to generate (noisy) estimation of the target location that are predicted using a Kalman filter. Multiple moving small ground robots can be used to observe animals using range sensors and binary decisions to indicate the presence/absence of a target [7]. The location of a target is corrected locally by using binary decisions collected from a wireless sensor network. Tracking is based on a penalized maximum likelihood framework to address the problem of a variable number of targets as animals enter (appear) and exit (disappear from) the environment. Multiple moving targets can be observed in a multi-region structured environment [43], with the assumption of prior knowledge on the densities of the locations of both targets and robots. Coordination of robots in the same region can be avoided, because they have the same information about the region and the targets within that region. This approach was extended to outdoor environments [45] for tracking multiple targets in regions with a high target-to-robot density ratio.

Using a flocking control algorithm, swarms of ground 
TABLE VI: Tasks relevant to the four control techniques. CT: Cooperative Tracking; CMOMMT: Cooperative Multi-robot Observation of Multiple Moving Targets; CSAT: Cooperative Search, Acquisition and Track; MPE: Multi-robot Pursuit Evasion.

\begin{tabular}{|c|c|}
\hline Control technique & Tasks \\
\hline CT & surveillance \\
\hline CMOMMT & patrolling and monitoring \\
\hline CSAT & situational awareness, search and rescue \\
\hline MPE & guarding, rescue in disaster areas, games \\
\hline
\end{tabular}

robots with omnidirectional range sensors have been used to track targets that avoid obstacles in a structured and cluttered environment [54], [55]. Flocks of robots split and merge into multiple smaller flocks that track a single target. Each robot is assumed to know the location of other robots and targets. A combination of UAV and UGV [52] has also been proposed for tracking mobile targets, where ground robots move in a structured and cluttered environment. They used range sensors with omnidirectional circular FOVs in a rectangular environment. Each new observation triggers an exchange of information between robots and causes a change in the behavior of the robot. The work was further extended to include detection errors in sensing and by minimizing energy consumption for sensor path-planning [50], [53].

\section{B. Cooperative Multi-robot Observation of Multiple Moving Targets (CMOMMT)}

The goal of CMOMMT is to dynamically position robots to maximize the collective time during which targets are observed, when the number of moving targets is larger than the number of robots. CMOMMT is used for monitoring, reconnaissance and patrolling tasks. The number of noncooperative targets with unknown locations is constant, and the environment is uncluttered and with regular shape. A target is assumed to be under observation when it is within the FOV of a robot. The problem is to maximize not only the number of targets under observation but also the duration of observation for each target.

CMOMMT is an NP-hard problem and was first proposed by Parker and Emmons [9]. To maximize the collective time of observation, robots operate in search or track mode. Mode switching is decided based on the presence of targets in the FOV of each robot. In the search mode, the robot aims at detecting targets. When targets are found the robot changes to the track mode and moves toward the center of mass of all the moving targets under its observation. When there are no more targets in its FOV, the robot switches back to search mode. Coordination is achieved by local force vectors that attract or repel robots [9], [60]: a robot is attracted by the targets to stay close enough for observing them and repelled by neighboring robots to avoid observation overlap.

Approximate CMOMMT (A-CMOMMT) includes weighted local force-vectors [59], [60] to reduce observation overlap of a single target by multiple robots to help increase the collective time of observation. Personality CMOMMT (P-CMOMMT) [61] addresses the problem that some targets may be observed for most of the time, while others could be completely unobserved. To make sure that all targets are observed, P-CMOMMT uses information entropy for the evaluation of the target observation diversity. To minimize the problem of losing a target from observation, Weighted CMOMMT (W-CMOMMT) assigns different priority weights to targets based on Broadcast of Local Eligibility (BLA) [63].

Behavioral CMOMMT (B-CMOMMT) [10], [62] includes a third mode of operation, the help mode. When a robot is loosing a target from its FOV, it broadcasts a help request to the other robots. The robots in search mode respond to these help requests by approaching the robot that is in need of help. B-CMOMMT also introduces targets tags in the coordination process to reduce overlap in target observation. B-CMOMMT can be improved with an extended Kohonen map for each robot to reach the target and an auction-based algorithm for cooperation [8].

Instead of using local force vectors and help calls, Formation-CMOMMT (F-CMOMMT) uses a flexible formation of robots [64]. Model-predictive control strategies [65] can also be used for CMOMMT but at the cost of a high computational complexity. The effects of degree of decentralization, speed of targets, and sensing range on collective observation of targets are analyzed in [66]. The work compares K-means clustering and hill-climbing algorithms, which are scalable in degree of decentralization, for achieving the objective of CMOMMT. The expected motion patterns of the targets can be exploited to observe each target for an equal amount of time [67].

The CMOMMT framework has been used for iceberg observation [68], [69]. The number of targets in the iceberg observation problem varies with time and the entry/exit points of these targets are unknown. The objective in such a problem is to minimize the time of initial contact with the newly generated targets.

The strengths of CMOMMT are its capability of switching between modes of operation (search and track) and of working under limited communication ranges. However, most CMOMMT approaches are based on uniform FOVs, observations with constant resolution and assume a sensor without errors. Moreover, as soon as a robot finds one or more targets in its FOV it starts its tracking mode, thus restricting the search of the remaining regions that may contain more targets. In addition to this, there is no situational awareness, as robots do not share their perceptions of the environment. For example, in search mode, robots work independently without any cooperation. For these reasons, CMOMMT is not suitable for real-world applications where the assumption of perfect sensors is unrealistic and situational awareness is needed.

\section{Cooperative Search, Acquisition and Track (CSAT)}

In CSAT, the problem is to continuously search for unknown targets and to track already located targets. CSAT integrates search and track modes, and these two modes alternate with respect to time or the level of uncertainty about the target location. CSAT assumes non-cooperative targets with unknown locations and uncluttered environment. Task assignment is 
used for the team of robots to search and track (observe) as many targets as possible. Each robot in CSAT purposely switches its mode repeatedly between search and track. Mode switching is decided using the uncertainty level in the location of a target. The robot tries to keep the location uncertainty of a detected target under a given bound throughout the mission.

Most of the work in CSAT uses aerial robots with a downward pointing camera for observation [1], [13], [14]. Unlike CMOMMT, in CSAT noisy sensors and measurement noise can be handled. A robot tracks only one target at a time and can switch to search mode even if it is successfully tracking a target. In CSAT, mode changes are frequent and a robot does not lock its operation in a given mode thus facilitating keeping a balance between search and track operations [14].

Targets are tracked only for a specified amount of time or until their states are adequately determined. Once a target is located accurately, its location is recorded and the target is temporarily left unobserved. The robot then starts searching for more targets thus causing a gradual increase in the uncertainty about the location of already located target. To keep this uncertainty under a given bound the robot quits the search mode, approaches the previously located target, and switches back to track mode. The motivation for a robot to switch its mode from tracking to search is the assumption that there is always at least one unknown target.

CSAT is used for search and rescue operations and situational awareness, i.e. the perception of the environment, the robots and the targets with respect to time, space or a predetermined event. Situational awareness may be in the form of search map that is updated by all the robots to aid the search process.

The first CSAT approach used a recursive Bayesian framework and a 2D grid containing a probability density function (PDF) of a target that guides the robot movement for search and maintains the information about the environment and target [1]. The robots use the PDF to share their perceptions of the environment and to decide on the mode switching. A tracking metric based on the covariance matrix of the target state [13] incorporates the growth of uncertainty on the target locations. Besides the covariance matrix, a multi-agent task assignment algorithm is used for mode switching [14], [71]. To integrate the conflicting objectives of target-search and target-track, an objective function is used that is based on the average value of information gained by the mission and that represents the number of targets detected and how well each detected target is tracked [70]. Different terms in the objective function control the importance given to detection and tracking. This approach depends on prior information about target distributions and lacks on-line path generation.

Existing CSAT approaches face the same problem as CMOMMT: a small number of targets may capture the attention of a robot (i.e. a robot frequently switches mode only for a small subset of targets in a specific region of the environment).

\section{Multi-robot Pursuit Evasion (MPE)}

Unlike in CT, CMOMMT and CSAT, in MPE targets can be evasive and can move faster than the robots. MPE approaches aim to observe (i.e. capture) targets only once. Therefore the problem in MPE is to minimize the time required to capture one or more evasive targets. Examples of MPE applications include monitoring exhibition areas [72], rescue operations in disaster areas [105] and mobile gaming [76], [106], [107]. Moreover, evading targets moving underwater in 3D can be captured using cooperative UUVs [19], [108], [109].

A distinguishing feature of pursuit-evasion (also known as adversarial search or hunting of targets [19], [108]) is the intelligence of the target that has full knowledge of the environment and is aware of the robot location and intent [110]. The robot and target motions are therefore inter-dependent as robots and targets compete with each other [31]. For this reason, motionplanning problems that arise in adversarial settings are related to a probabilistic game theoretical framework.

While single-target pursuit-evasion has been an active topic of research for the last two decades (see review in [110]), only a few works consider observation of multiple moving targets by multiple moving robots, i.e. the Multi-robot Pursuit Evasion (MPE) problem [20], [111]. Two main MPE variations exist [110]. In the first variation, a robot associates itself with a captured target and maintains this association until the mission ends [75] (target tracking for only $M$ targets). In the second variation, a target is removed from the mission as soon as it is detected/captured [74], [108] and the robot that captured this target continues looking for other targets.

Several works assume unstructured environments. A distributed approach based on hierarchical decomposition algorithm for differential game theory was used for UAVs [76]. The deterministic model of the sensor (with no sensing errors) makes the approach less attractive and applicable. Targets can be captured using a centralized MPE algorithm with taskscheduling heuristics that assign robots to different parts of the environment, which was partitioned using Voronoi diagrams [77]. Economics-based negotiation and auction mechanisms were used to assign multiple robots to the targets [78], with the objective of maximizing the probability of capture while minimizing the time to capture. In order to achieve this objective, the robots used dynamic coalition formation for planning their paths.

Other MPE works consider irregularly shaped, structured and planar environments with obstacles for area coverage using range sensors with conical FOVs [72]. These works use a team of mobile ground robots and a deterministic error-free sensor model to guarantee the capture of the targets. A greedy decentralized approach that employs a heterogeneous team of UAVs and UGVs was used to minimize the capture time of targets in [20]. A distinctive characteristic of this approach is the modeling of detection errors in the sensing process.

\section{CONCLUSIONS AND FUture DiRECTIONS}

In this review, we organized, critically discussed and compared works from the last 20 years in the area of cooperative mobile robots for observing multiple moving targets. We identified five factors that affect the design and performance of cooperative mobile robots. It is important to notice that most related works on the topic are based on simulation and lab 
studies, which may sometimes not consider constraints and challenges from real-life applications.

We conclude the paper with a list of important future directions of research and development in this area, which include dealing with the limitations of wireless networks, the coordination with human operators, safety and privacy issues.

The development of protocols, algorithms and applications that can deal with various network limitations will advance the applications of mobile robots. Delay tolerant networks must be designed to degrade gracefully when the communication is slow, unavailable and intermittent. Moreover, interoperability is desirable to enable mobile robots to communicate with internet-based services in the cloud and to other interconnected devices. This will enhance the maintenance, operations, and updates of mobile robot systems [24].

The level of cognition and autonomy, i.e., the ability to plan and execute tasks in response to the high level commands, is increasing rapidly and there is great potential for cooperative mobile robots observing moving targets. The potential impact of mobile robots will depend not only on the coordination among robots themselves but also on their joint coordination with human operators. Existing works are limited in investigating such collaboration. While automated systems without the interaction with humans may be faster and more efficient in dynamic and time-critical environments, human operators can assist with their knowledge-based reasoning [71] and to dynamically assign different targets or roles to robots. Studies should examine how an operator interacts with decentralized robots to help develop novel human computer/automation interaction methods to assist human operators in control tasks with multiple robots.

The emergence of autonomous robotics also introduces ethical, legal and societal issues. Autonomous and networked mobile robots in unknown environments may (will) malfunction thus leading to safety issues. While safety was traditionally implemented by isolating the robot's operating space with physical barriers, the use of cooperative mobile robots outside a manufacturing plant in a shared robots-human space creates new safety challenges. Safety must be designed for a multirobot system and tested according to well-defined standards that have to be developed. Also, methods to verify and certify safety in applications where humans and robots interact or share the same physical space are needed.

Robots can capture sensitive private data and may pose a privacy threat. The protection of sensitive data must be guaranteed with appropriate access rights to potentially sensitive data held by robots. Moreover, the use of networking for robot coordination facilitates unauthorized access to information and attacks that can cause physical disruptions in services provided by the robots, which can be physically attacked or hacked [112]. IT-security mechanisms should therefore prevent cyberattacks. Moreover, functionalities able to detect physically damaged or hacked robots autonomously during runtime shall be provided.

\section{ACKNOWLEDGEMENTS}

A. Khan was supported in part by the EACEA Agency of the European Commission under EMJD ICE (FPA no 2010-
0012). The work was supported in part by the ERDF, KWF, and BABEG under grant KWF-20214/24272/36084 (SINUS) and was performed in the research cluster Lakeside Labs $\mathrm{GmbH}$ and in the Centre for Intelligent Sensing, Queen Mary University of London.

\section{REFERENCES}

[1] T. Furukawa, F. Bourgault, B. Lavis, and H. F. Durrant-Whyte, "Recursive Bayesian search-and-tracking using coordinated UAVs for lost targets," in Proceedings of the IEEE International Conference on Robotics and Automation, 2006, pp. 2521-2526.

[2] C. Lecher, How will drones change sports, 2014 [Online]. Available: http://www.popsci.com/article/technology/ how-will-drones-change-sports

[3] A. M. Khaleghi, D. Xu, Z. Wang, M. Li, A. Lobos, J. Liu, and Y.-J. Son, "A DDDAMS-based planning and control framework for surveillance and crowd control via UAVs and UGVs," Expert Systems with Applications, vol. 40, no. 18, pp. 7168-7183, 2013.

[4] B. Livingston, The role of UAVs/MAVs in domestic surveillance and control. [Online]. Available: http://www.rense.com/general47/uav.htm

[5] D. J. Pack, P. DeLima, G. J. Toussaint, and G. York, "Cooperative control of UAVs for localization of intermittently emitting mobile targets," IEEE Transactions on Systems, Man, and Cybernetics, Part B: Cybernetics, vol. 39, no. 4, pp. 959-970, 2009.

[6] A. Rodriguez, J. J. Negro1, M. Mulero1, C. Rodriguez, J. HernandezPliego, and J. Bustamante, "The eye in the sky: Combined use of unmanned aerial systems and GPS data loggers for ecological research and conservation of small birds," PLoS ONE, vol. 7, no. 12, pp. 1-6, 2012.

[7] N. Katenkaa, E. Levinab, and G. Michailidis, "Tracking multiple targets using binary decisions from wireless sensor networks," Journal of The American Statistical Association, vol. 108, no. 502, pp. 398-410, 2013.

[8] A. M. Elmogy and F. O. Karray, "Cooperative multi-target tracking using multi sensor network," International Journal on Smart Sensing and Intelligent Systems, vol. 1, no. 3, pp. 716-734, 2008.

[9] L. E. Parker and B. A. Emmons, "Cooperative multi-robot observation of multiple moving targets," in Proceedings of the IEEE International Conference on Robotics and Automation, 1997, pp. 2082-2089.

[10] A. Kolling and C. Stefano, "Multirobot cooperation for surveillance of multiple moving targets - a new behavioral approach," in Proceedings of the IEEE International Conference on Robotics and Automation, 2006, pp. 1311-1316.

[11] N. Michael, D. Mellinger, Q. Lindsey, and V. Kumar, "The GRASP multiple micro UAV testbed," IEEE Robotics and Automation Magazine, vol. 17, no. 3, pp. 56-65, 2010.

[12] V. Kumar and N. Michael, "Opportunities and challenges with autonomous micro aerial vehicles," International Journal of Robotics Research, vol. 31, no. 11, pp. 1279-1291, 2012.

[13] E. W. Frew and J. Elston, "Target assignment for integrated search and tracking by active robot networks," in Proceedings of the IEEE International Conference on Robotics and Automation, 2008, pp. 2354 2359.

[14] J. P. How, C. Fraser, K. C. Kulling, L. F. Bertuccelli, O. Toupet, L. Brunet, A. Bachrach, and N. Roy, "Increasing autonomy of UAVs," IEEE Robotics and Automation Magazine, vol. 16, no. 2, pp. 43-51, 2009.

[15] A. Wallar, E. Plaku, and D. A. Sofge, "Reactive motion planning for unmanned aerial surveillance of risk-sensitive areas," IEEE Transactions on Automation Science and Engineering, vol. 12, no. 3, pp. 969-980, 2015.

[16] G. Han, C. Zhang, L. Shu, N. Sun, and Q. Li, "A survey on deployment algorithms in underwater acoustic sensor networks," International Journal of Distributed Sensor Networks, vol. 2013, pp. 1-11, 2013.

[17] Z. Zeng, L. Lian, K. Sammut, F. He, Y. Tang, and A. Lammas, "A survey on path planning for persistent autonomy of autonomous underwater vehicles," Ocean Engineering, vol. 110, no. A, pp. 303313,2015

[18] Z. Zeng, A. Lammas, K. Sammut, F. He, Y. Tang, and Q. Ji, "Path planning for rendezvous of multiple AUVs operating in a variable ocean ," in Proceedings of the IEEE International Conference on Cyber Technology in Automation, Control and Intelligent Systems, 2014, pp. $451-456$.

[19] C. Xiang and Z. Daqi, "A survey of cooperative hunting control algorithms for multi-AUV systems ," in Proceedings of the Chinese Control Conference, 2013, pp. 5791-5795. 
[20] R. Vidal, O. Shakernia, H. J. Kim, D. H. Shim, and S. Sastry, "Probabilistic pursuit-evasion games: Theory, implementation, and experimental evaluation," IEEE Transactions on Robotics and Automation, vol. 18 , no. 5 , pp. 262-269, 2002.

[21] J. Chen, X. Zhang, B. Xin, and H. Fang, "Coordination between unmanned aerial and ground vehicles: A taxonomy and optimization perspective," IEEE Transactions on Cybernetics, vol. 46, no. 4, pp. 959-972, 2016.

[22] M. Aranda, G. Lopez-Nicol, C. Sagües, and Y. Mezouar, "Formation control of mobile robots using multiple aerial cameras," IEEE Transactions on Robotics, vol. 31, no. 4, pp. 1064-1071, 2015.

[23] A. Farinelli, L. Iocchi, and D. Nardi, "Multi-robot systems: A classification focused on coordination," IEEE Transactions on Systems, Man, and Cybernetics, Part B: Cybernetics, vol. 34, no. 5, pp. 2015-2028, 2004.

[24] B. Kehoe, S. Patil, P. Abbeel, and K. Goldberg, "A survey of research on cloud robotics and automation," IEEE Transactions on Automation, Science and Engineering, vol. 12, no. 2, pp. 398-407, 2015.

[25] F. Bonin-Font, A. Ortiz, and G. Oliver, "Visual navigation for mobile robots: A survey," Journal of Intelligent Robotic Systems, vol. 53, no. 3 , pp. 263-296, 2008.

[26] M. Elbanhawi and M. Simic, "Sampling-based robot motion planning: A review," IEEE Access, vol. 2, pp. 56-77, 2014.

[27] C. Goerzen, Z. Kong, and B. Mettler, "A survey of motion planning algorithms from the perspective of autonomous UAV guidance," Journal of Intelligent and Robotic Systems, vol. 100, no. 1, pp. 57-65, 2010.

[28] T. Arai, E. Pagello, and L. E. Parker, "Guest editorial: Advances in multirobot systems," IEEE Transactions on Automation, Science and Engineering, vol. 18, no. 5, pp. 655-661, 2002.

[29] Y. U. Cao, A. S. Fukunaga, and A. B. Kahng, "Cooperative mobile robotics: Antecedents and directions," Autonomous Robots, vol. 4, no. 1, pp. 1-13, 1997.

[30] M. B. Dias, R. Zlot, N. Kalra, and A. Stentz, "Market-based multirobot coordination: A survey and analysis," Proceedings of the IEEE, vol. 94, no. 7, pp. 1257-1270, 2006.

[31] Z. Yan, N. Jouandeau1, and A. A. Cherif, "A survey and analysis of multi-robot coordination," International Journal of Advanced Robotic Systems, vol. 10, pp. 1-18, 2013.

[32] C. Robin and S. Lacroix, "Multi-robot target detection and tracking: taxonomy and survey," Autonomous Robots, vol. 12, no. 3, pp. 1-32, 2015.

[33] G. A. Hollinger, S. Yerramalli, S. Singh, U. Mitra, and G. S. Sukhatme, "Distributed data fusion for multirobot search," IEEE Transactions on Robotics, vol. 31, no. 1, pp. 55-66, 2015.

[34] G. A. Hollinger and S. Singh, "Multirobot coordination with periodic connectivity: Theory and experiments," IEEE Transactions on Robotics, vol. 28, no. 4, pp. 967-973, 2012.

[35] K. Zhou and S. I. Roumeliotis, "Multirobot active target tracking with combinations of relative observations," IEEE Transactions on Robotics, vol. 27, no. 4, pp. 678-695, 2011.

[36] R. K. Williams and G. S. Sukhatme, "Observability in topologyconstrained multi-robot target tracking," in Proceedings of the IEEE International Conference on Robotics and Automation, 2015, pp. 17951801

[37] A. Macwan, J. Vilela, G. Nejat, and B. Benhabib, "A multirobot pathplanning strategy for autonomous wilderness search and rescue," IEEE Transactions on Cybernetics, vol. 45, no. 9, pp. 1784-1797, 2015.

[38] Y. Cai, N. de Freitas, and J. J. Little, "Robust visual tracking for multiple targets," in Procceedings of the European Conference on Computer Vision, 2006, pp. 107-118.

[39] A. Milan, S. Roth, and K. Schindler, "Continuous energy minimization for multitarget tracking," IEEE Transactions on Pattern Analysis and Machine Intelligence, vol. 36, no. 1, pp. 58-72, 2014.

[40] J. Kim and J. L. Crassidis, "UAV path planning for maximum visibility of ground targets in an urban area," in Proceedings of the IEEE Conference on Information Fusion, 2010, pp. 1-7.

[41] P. K. Yadav and W. Meng, "Mobile targets localization in a field area using moving Gaussian peaks and probability map," in Proceedings of the IEEE International Conference on Control and Automation, 2014, pp. 416-421.

[42] J. Hu, L. Xie, J. Xu, and Z. Xu, "Multi-agent cooperative target search," Sensors, vol. 14, no. 6, pp. 9408-9428, 2014.

[43] B. Jung and G. S. Sukhatme, "A generalized region-based approach for multi-target tracking in outdoor environments," in Proceedings of the IEEE International Conference on Intelligent Robots and Systems, 2002, pp. 2764-2769.
[44] P. Tokekar, V. Isler, and A. Franchi, "Multi-target visual tracking with aerial robots," in Proceedings of the IEEE International Conference on Intelligent Robots and Systems, 2014, pp. 3067-3072.

[45] B. Jung and G. S. Sukhatme, "A region-based approach for cooperative multi-target tracking in a structured environment," in Proceedings of the IEEE International Conference on Intelligent Robots and Systems, 2004, pp. 2189-2195.

[46] B. Jung and G. Sukhatme, "A region-based approach for cooperative multi-target tracking in a structured environment," in Proceedings of International Symposium on Distributed Autonomous Robotic Systems, 2006, pp. 81-90.

[47] T. H. Chung, V. Gupta, J. W. Burdick, and R. M. Murray, "On a decentralized active sensing strategy using mobile sensor platforms in a network," in Proceedings of the IEEE Conference on Decision and Control, 2004, pp. 1914-1919.

[48] A. Sinha, T. Kirubarajan, and Y. Bar-Shalom, "Autonomous ground target tracking by multiple cooperative UAVs," in Proceedings of the IEEE Aerospace Conference, 2005, pp. 1-9.

[49] F. Morbidi and G. L. Mariottini, "Active target tracking and cooperative localization for teams of aerial vehicles," IEEE Transactions on Control Systems Technology, vol. 21, no. 5, pp. 1694-1707, 2013.

[50] D. Tolic and R. Fierro, "Adaptive sampling for tracking in pursuitevasion games," in Procceedings of the IEEE International Symposium on Intelligent Control, 2011, pp. 179-184.

[51] S. Kamath, E. Meisner, and V. Isler, "Triangulation based multi target tracking with mobile sensor networks," in Proceedings of the IEEE International Conference on Robotics and Automation, 2007, pp. 32833288.

[52] D. Tolic, R. Fierro, and S. Ferrari, "Cooperative multi-target tracking via hybrid modeling and geometric optimization," in Proceedings of the Mediterranean Conference on Control and Automation, 2009, pp. $440-445$

[53] S. Ferrari, R. Fierro, and D. Tolic, "A geometric optimization approach to tracking maneuvering targets using a heterogeneous mobile sensor network," in Proceedings of the IEEE Conference on Decision and Control, 2009, pp. 1080-1087.

[54] H. M. Laa and W. Shengb, "Dynamic target tracking and observing in a mobile sensor network," Robotics and Autonomous Systems, vol. 60, no. 7, pp. 996-1009, 2012.

[55] G. Lee, N. Y. Chong, and H. Christensen, "Tracking multiple moving targets with swarms of mobile robots," Intelligent Service Robotics, vol. 3, no. 2, pp. 61-72, 2010.

[56] Z. Tang and Ü. Özgüner, "Motion planning for multitarget surveillance with mobile sensor agents," IEEE Transactions on Robotics, vol. 21, no. 5, pp. 898-908, 2005.

[57] Y. Fu, Q. Ling, and Z. Tian, "Distributed sensor allocation for multitarget tracking in wireless sensor networks," IEEE Transactions on Aerospace and Electronic Systems, vol. 48, no. 4, pp. 3538-3553, 2012.

[58] Y. Fu and L. Yang, "Sensor mobility control for multitarget tracking in mobile sensor networks," International Journal of Distributed Sensor Networks, vol. 2014, no. 1, pp. 1-15, 2014.

[59] L. E. Parker and C. Touzet, "Multi-robot learning in a cooperative observation task," in Distributed Autonomous Robotic Systems 4, L. E. Parker, G. Bekey, and J. Barhen, Eds. Springer Japan, 2000, pp. 391-401.

[60] L. E. Parker, "Distributed algorithms for multi-robot observation of multiple moving targets," Autonomous Robots, vol. 12, no. 3, pp. 231255, 2002

[61] Y. Ding, M. Zhu, Y. He, and J. Jiang, "P-CMOMMT algorithm for the cooperative multi-robot observation of multiple moving targets," in Proceedings of the 6th World Congress on Intelligent Control and Automation, 2006, pp. 9267-9271.

[62] A. Kolling and S. Carpin, "Cooperative observation of multiple moving targets: an algorithm and its formalization," The International Journal of Robotics Research, vol. 26, no. 9, pp. 935-953, 2007.

[63] B. B. Werger and M. J. Mataric, "Broadcast of local eligibility for multi-target observation," in Distributed Autonomous Robotic Systems 4, L. E. Parker, G. Bekey, and J. Barhen, Eds. Springer Japan, 2000, pp. $347-356$

[64] Y. Ding and Y. He, "Flexible formation of the multi-robot system and its application on cmommt problem," in Proceedings of the International Asia Conference on Informatics in Control, Automation and Robotics, 2010, pp. 377-382.

[65] J. Kuhn, C. Reinl, and O. von Stryk, "Predictive control for multi-robot observation of multiple moving targets based on discrete-continuous linear models," in Proceedings of the 18th World Congress of the 
International Federation of Automatic Control (IFAC), 2011, pp. 257262.

[66] S. Luke, K. Sullivan, L. Panait, and G. Balan, "Tunably decentralized algorithms for cooperative target observation," in Proceedings of the Conference on Autonomous Agents and Multi-Agent Systems (AAMAS), 2005, pp. 911-917.

[67] J. Banfi, J. Guzzi, A. Giusti, L. Gambardella, and G. A. D. Caro, "Fair multi-target tracking in cooperative multi-robot systems," in Proceedings of the IEEE International Conference on Robotics and Automation, 2015, pp. 5411-5418.

[68] R. A. Coogle and A. M. Howard, "The iceberg observation problem: Using multiple agents to monitor and observe ablating target sources," in Proceedings of the IEEE International Conference on Systems, Man, and Cybernetics, 2013, pp. 1660-1665.

[69] R. A. Coogle and A. Howard, "Robotic resource allocation for the observation of oblating target sources," in Proceedings of the IEEE International Conference on Systems, Man, and Cybernetics, 2014, pp. 4002-4007.

[70] R. R. Pitre, X. R. Li, and R. Delbalzo, "UAV route planning for joint search and track missions: An information-value approach," IEEE Transactions on Aerospace and Electronic Systems, vol. 48, no. 3, pp. 2551-2565, 2012.

[71] M. L. Cummings, J. P. How, A. Whitten, and O. Toupet, "The impact of human-automation collaboration in decentralized multiple unmanned vehicle control," Proceedings of the IEEE, vol. 100, no. 3, pp. 660-671, 2012.

[72] J. W. Durham, A. Franchi, and F. Bullo, "Distributed pursuit-evasion without mapping or global localization via local frontiers," Autonomous Robots, vol. 32, pp. 81-95, 2012.

[73] S. Jin and Z. Qu, "A heuristic task scheduling for multi-pursuer multievader games," in Proceedings of the IEEE International Conference on Information and Automation, 2011, pp. 528-533.

[74] K. H. Wray and B. B. Thompson, "An application of multiagent learning in highly dynamic environments," in Proceedings of the AAAI Workshop on Multiagent Interaction without Prior Coordination (MIPC), 2014, pp. 1-7.

[75] J. A. Alcazar, "A simple approach to multi-predator multi-prey pursuit domain," in Unifying Themes in Complex Systems, A. A. Minai, D. Braha, and Y. Bar-Yam, Eds. Springer Berlin Heidelberg, 2011, pp. 2-9.

[76] J. Ge, L. Tang, J. Reimann, and G. Vachtsevanos, "Hierarchical decomposition approach for pursuit-evasion differential game with multiple players," in Procceedings of the IEEE Aerospace Conference, 2006, pp. 1-7.

[77] S. Jin and Z. Qu, "A heuristic task scheduling for multi-pursuer multievader games," in Proceedings of the IEEE International Conference on Information and Automation, 2011, pp. 528-533.

[78] Z.-S. Cai, L.-N. Sun, H.-B. Gao, P.-C. Zhou, S.-H. Piao, and Q.C. Huang, "Multi-robot cooperative pursuit based on task bundle auctions," in Intelligent Robotics and Applications, C. Xiong, Y. Huang, Y. Xiong, and H. Liu, Eds. Springer Berlin Heidelberg, 2008, vol. 5314 , pp. 235-244.

[79] J. Su and W. Xie, "Motion planning and coordination for robot systems based on representation space," IEEE Transactions on Systems, Man, and Cybernetics-Part B: Cybernetics, vol. 41, no. 1, pp. 248-259, 2011.

[80] M. Schwager, B. J. Julian, M. Angermann, and D. Rus, "Eyes in the sky: decentralized control for the deployment of robotic camera networks," Proceedings of the IEEE, vol. 99, no. 9, pp. 1541-1561, 2011.

[81] S. Yahyanejad and B. Rinner, "A fast and mobile system for registration of low-altitude visual and thermal aerial images using multiple smallscale uavs," ISPRS Journal of Photogrammetry and Remote Sensing, vol. 104, pp. 189-202, 2015.

[82] M. P. Kolba, W. R. Scott, and L. M. Collins, "A Framework for Information-Based Sensor Management for the Detection of Static Targets," IEEE Transactions on Systems, Man and Cybernetics, Part A: Systems and Humans, vol. 41, no. 1, pp. 105-120, 2011.

[83] A. Khan, B. Rinner, and A. Cavallaro, "Multi-scale observation of multiple moving targets using micro aerial vehicles," in Proceedings of the IEEE International Conference on Intelligent Robots and Systems, 2015, pp. 1-6.

[84] P. Dames, P. Tokekar, and V. Kumar, "Detecting, Localizing, and tracking an unknown number of moving targets using a team of mobile robots," in International Symposium on Robotics Research, 2015, pp. $1-6$.
[85] Z. Sunberg, S. Chakravorty, and R. S. Erwin, "Information space receding horizon control for multisensor tasking problems," IEEE Transactions on Cybernetics, vol. 46, no. 6, pp. 1325-1336, 2016.

[86] D. Carevic, "Detection and tracking of underwater targets using directional sensors," in Proceedings of the 3rd International Conference on Intelligent Sensors, Sensor Networks and Information., 2007, pp. 143-148.

[87] J. Haugen and L. Imsland, "Monitoring moving objects using aerial mobile sensors," IEEE Transactions on Control Systems Technology, vol. 24, no. 2, pp. 475-486, 2016.

[88] W. Zhao, Q. Meng, and P. W. H. Chung, "A heuristic distributed task allocation method for multivehicle multitask problems and its application to search and rescue scenario," IEEE Transactions on Cybernetics, vol. 46, no. 4, pp. 902-915, 2016.

[89] A. Elfes, "Occupancy grids: A stochastic spatial representation for active robot perception," in Proceedings of Conference on Uncertainty in Artificial Intelligence, 1990, pp. 60-70.

[90] X. Yi, A. Zhu, S. X. Yang, and C. Luo, "A bio-inspired approach to task assignment of swarm robots in 3-D dynamic environments," IEEE Transactions on Cybernetics, pp. 1-10, 2016.

[91] A. Renzaglia, L. Doitsidis, A. Martinelli, and E. Kosmatopoulos, "MultiRobot 3D coverage of unknown areas," International Journal of Robotics Research, SAGE Publications, vol. 31, no. 6, pp. 738-752, 2012.

[92] S. Oh, L. Schenato, P. Chen, and S. Sastry, "Tracking and coordination of multiple agents using sensor networks: System design, algorithms and experiments," Proceedings of the IEEE, vol. 95, no. 1, pp. 234-253, 2007.

[93] A. Khan, E. Yanmaz, and B. Rinner, "Information merging in multiUAV cooperative search," in Proceedings of the IEEE International Conference on Robotics and Automation, 2014, pp. 3122-3129.

[94] M. Schranz and B. Rinner, "Resource-aware dynamic clustering utilizing state estimation in visual sensor networks," Sensors and Transducers, vol. 191, no. 8, pp. 28-39, 2015.

[95] D. Scaramuzza, R. Siegwart, and A. Martinelli, "A robust descriptor for tracking vertical lines in omnidirectional images and its use in mobile robotics," The International Journal of Robotics Research, vol. 28, no. 2, pp. 149-171, 2009.

[96] C. Changmook, S. SeungBeum, R. Chi-won, K. Yeonsik, K. Sungchul, L. Jung-yup, and H. Chang-soo, "Sensor fusion-based line detection for unmanned navigation," in Proceedings of the IEEE Intelligent Vehicle Symposium, 2010, pp. 191-196.

[97] A. Koubêa and J. M. de Dios, Cooperative robots and sensor networks, ser. Studies in Computational Intelligence. Springer International Publishing, 2015, vol. 604.

[98] C. Piciarelli, L. Esterle, A. Khan, B. Rinner, and G. L. Foresti, "Dynamic reconfiguration in camera networks: a short survey," IEEE Transactions on Circuits and Systems for Video Technology, vol. 26, no. 5, pp. 965-977, 2016.

[99] T. Andre, K. A. Hummel, A. P. Schoellig, E. Yanmaz, M. Asadpour, C. Bettstetter, P. Grippa, H. Hellwagner, S. Sand, and S. Zhang, "Application-driven design of aerial communication networks," IEEE Communications Magazine, vol. 52, no. 5, pp. 129-137, 2014.

[100] S. Yoon and C. Qiao, "Cooperative search and survey using autonomous underwater vehicles (AUVs)," IEEE Transactions on Parallel and Distributed Systems, vol. 22, no. 3, pp. 364-379, 2011.

[101] L. D. Stone, R. L. Streit, T. L. Corwin, and K. L. Bell, "Bayesian multiple target tracking." Artech House, 2013.

[102] T. Theodoridis and $\mathrm{H}$. Hu, "Toward intelligent security robots: A survey," IEEE Transactions on Systems, Man, and Cybernetics - Part C: Applications and reviews, vol. 42, no. 6, pp. 1219-1230, 2012

[103] N. Farmani, L. Sun, and D. Pack, "An optimal sensor management technique for unmanned aerial vehicles tracking multiple mobile ground targets," in International Conference on Unmanned Aircraft Systems (ICUAS), 2014, pp. 570-576.

[104] Y. Wang and A. Cavallaro, "Prioritized target tracking with active collaborative cameras," in Proceedings of the IEEE International Conference on Advanced Signal and Video based Surveillance (AVSS), 2016.

[105] Z. Wang and S. Zlatanova, in Progress in location-based services, ser. Lecture Notes in Geoinformation and Cartography. Springer Berlin Heidelberg, 2013, ch. Taxonomy of navigation for first responders, pp. 297-315.

[106] P. Kachroo, S. A. Shedied, J. S. Bay, and H. Vanlandingham, "Dynamic programming solution for a class of pursuit evasion problems: The herding problem," IEEE Transactions on Systems, Man, and Cybernet- 
ics - part C: Applications and Reviews, vol. 31, no. 1, pp. 35-41, 2001.

[107] G. N. Yannakakis, J. Levine, and J. Hallam, "Emerging cooperation with minimal effort: rewarding over mimicking," IEEE Transactions on Evolutionary Computation, vol. 11, no. 3, pp. 382-396, 2007.

[108] J. Ni and S. X. Yang, "Bioinspired Neural Network for Real-Time Cooperative Hunting by Multirobots in Unknown Environments," IEEE Transactions on Neural Networks, vol. 22, no. 12, pp. 2062-2077, 2011.

[109] D. Zhu, H. Huang, and S. X. Yang, "Dynamic task assignment and path planning of multi-AUV system based on an improved self-organizing map and velocity synthesis method in three-dimensional underwater workspace," IEEE Transactions on Cybernetics, vol. 43, no. 2, pp. 504-514, 2013.

[110] T. H. Chung, G. A. Hollinger, and V. Isler, "Search and pursuit-evasion in mobile robotics: A survey," Autonomous Robots, vol. 31, no. 4, pp. 299-316, 2011.

[111] R. Borie, S. Koenig, and C. Tovey, "Section 9.5: Pursuit-evasion problems," in Handbook of Graph Theory, J. Gross, J. Yellen, and P. Zhang, Eds. Chapman and Hall/CRC, 2013, pp. 1145-1165.

[112] T. Humphreys, Cockrell School researchers demonstrate first successful spoofing of UAVs, 2012. [Online]. Available: http://www.engr.utexas. edu/features/humphreysspoofing

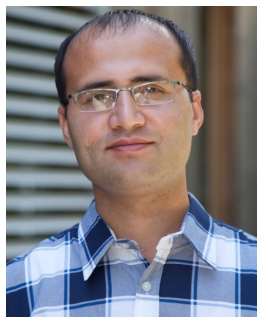

Asif Khan is Assistant Professor with Ghulam Ishaq Khan (GIK) Institute of Engineering Sciences and Technology, Swabi, Pakistan. He received M.S. degree in Electronics and Communication Engineering from Myongji University, Yongin, South Korea in 2009 and Ph.D. degree in Interactive and Cognitive Environments jointly awarded by the three universities: Alpen-Adria-Universität Klagenfurt, Klagenfurt, Austria, Queen Mary University of London, London, UK, and Università degli Studi di Genova, Genova, Italy in 2015. He was awarded full scholarship by Higher Education Commission of Pakistan for his M.S. and Erasmus Mundus scholarship by European Commission for his Ph.D. His main research interests include multi-robot systems, unmanned aerial vehicles and cooperative search.

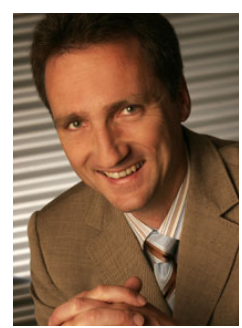

Bernhard Rinner (Senior Member, IEEE) received the M.Sc. and Ph.D. degrees in Telematics from Graz University of Technology, Austria in 1993 and 1996, respectively. He is full professor and chair of pervasive computing at Klagenfurt University. He held research positions with Graz University of Technology from 1993 to 2007 and with the University of Texas at Austin, from 1998 to 1999 His current research interests include embedded computer vision, aerial robotics, sensor networks and pervasive computing. He is associate editor of the Ad Hoc Networks Journal and EURASIP Journal on Embedded Systems and is member of the board of the Austrian Science Fund.

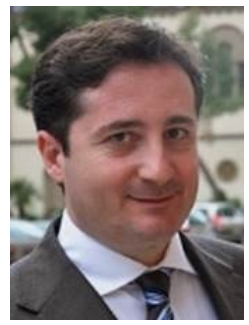

Andrea Cavallaro is Professor of Multimedia Signal Processing and Director of the Centre for Intelligent Sensing at Queen Mary University of London, UK. He received his Ph.D. in Electrical Engineering from the Swiss Federal Institute of Technology (EPFL), Lausanne, in 2002. He was a Research Fellow with British Telecommunications (BT) in 2004/2005 and was awarded the Royal Academy of Engineering teaching Prize in 2007; three student paper awards on target tracking and perceptually sensitive coding at IEEE ICASSP in 2005, 2007 and 2009; and the best paper award at IEEE AVSS 2009. Prof. Cavallaro is Associate Editor for the IEEE Transactions on Circuits and Systems for Video Technology and member of the editorial board of IEEE Multimedia. $\mathrm{He}$ is a past Area Editor for IEEE Signal Processing Magazine and a past Associate Editor for the IEEE Transactions on Image Processing, IEEE Transactions on Multimedia, IEEE Transactions on Signal Processing, and IEEE Signal Processing Magazine. He has published over 160 journal and conference papers, one monograph on Video tracking (2011,Wiley) and three edited books: Multi-camera networks (2009, Elsevier); Analysis, retrieval and delivery of multimedia content (2012, Springer); and Intelligent multimedia surveillance (2013, Springer). 